\title{
Simultaneous observations of reflection echoes and refractive index gradient in the troposphere and lower stratosphere
}

\author{
T. Tsuda, P. T. May, T. Sato, S. Kato, and S. Fukao \\ Radio Atmospheric Science Center, Kyoto University, Uji, Kyoto, Japan
}

(Received August 7, 1987; revised February 17, 1988; accepted April 11, 1988.)

\begin{abstract}
We have studied some characteristics of clear air echoes in the lower stratosphere and troposphere from simultaneous observations of vertical echo power and temperature profiles. The vertical echo power has been oversampled every $75 \mathrm{~m}$ with a height resolution of $150 \mathrm{~m}$ by the middle and upper atmosphere (MU) radar $\left(35^{\circ} \mathrm{N}, 136^{\circ} \mathrm{E}\right)$. During the radar observations a radiosonde was launched at the MU radar site in order to measure temperature, humidity, and pressure with a height resolution of a few tens of meters, from which the mean gradient of generalized potential refractive index, $M$, was determined. In the lower troposphere (below $10 \mathrm{~km}$ altitude), $M$ is enhanced owing to humidity by about $10-20 \mathrm{~dB}$, and its fine structure is mainly determined by the vertical gradient of humidity. The relatively large time-height variation of tropospheric echo power seems to be attributed to rapid changes in the humidity profile. On the other hand, in the upper troposphere (above $10 \mathrm{~km}$ altitude) and stratosphere the vertical structure of $M$ is mainly determined by the Brunt-Väisälä frequency and air density, where the former determines fine vertical structure of $M$ and the latter the gradual decrease in $M$ with a scale height of about $7 \mathrm{~km}$. The measured $M^{2}$ profile agrees well with the vertical echo power profile down to the radar height resolution of $150 \mathrm{~m}$. That is, the vertical structure of the reflection coefficient is mainly determined by $M^{2}$, and therefore the energy density of 3-m scale fluctuations $E(2 k)$ seems to be distributed uniformly with height. The vertical spacing of intense reflection layers usually ranges from $500 \mathrm{~m}$ to a few kilometers, which corresponds to the dominant vertical scale of fluctuations in the Brunt-Väisälä frequency profile. The vertical distribution of intense reflection layers seems to be explained by a predominance of a saturated vertical wave number spectrum of gravity waves with a slope of -3 and a dominant vertical scale of a few kilometers.
\end{abstract}

\section{INTRODUCTION}

MST radars operated at VHF and UHF utilize a physical principle that radio waves in these frequency bands are scattered and/or reflected by fluctuations in the radio refractive index of the atmosphere. They are extensively used for the monitoring of the three components of the wind field and turbulence parameters in the troposphere and middle atmosphere. It is widely accepted that for VHF radar observations isotropic turbulent scattering is dominant in the oblique directions [VanZandt et al., 1978], and reflection from stratified layers becomes dominant in the vertical direction [Gage and Green, 1978; Röttger and Liu, 1978; Gage and Balsley, 1980; Röttger, 1980]. By using the unique versatility of the middle and upper atmosphere (MU) radar [Kato et al., 1984; Fukao et

Copyright 1988 by the American Geophysical Union.

Paper number 850300 .

0048-6604/88/008S-0300\$08.00 al., 1985a, b], Tsuda et al. [1986] have detected a large aspect sensitivity of stratospheric echoes at zenith angles up to $10^{\circ}$ which seems to be caused by Fresnel reflection. Various models modified from the Fresnel reflection mechanism, such as diffuse reflection [Röttger, 1980] or Fresnel scattering [Hocking and Röttger, 1983; Gage et al., 1985], have been proposed to describe the actual behavior of reflection echoes in the lower atmosphere.

This study is mainly concerned with the characteristics of reflection echoes observed from the vertical direction in the troposphere and lower stratosphere in September 1986 by the MU radar. We present a profile of the refractive index gradient simultaneously measured by a radiosonde launched at the MU radar site and discuss dominant mechanisms that determine the refractive index gradients in the wet (below $10 \mathrm{~km}$ ) and dry (above $10 \mathrm{~km}$ ) atmospheres separately. We also discuss the effects of gravity waves on the reflection layers in the dry atmosphere in terms of a saturated vertical wave number spectrum of temperature perturbations. 


\section{REFRACTIVE INDEX GRADIENT}

The refractive index $n$ in the lower atmosphere is expressed as follows:

$$
n-1=0.37 e / T^{2}+77.6 \times 10^{-6} p / T
$$

where $e, p$, and $T$ are partial pressure of water vapor in millibars, atmospheric pressure, and temperature, respectively [e.g., Bean and Dutton, 1966]. The first and second terms are usually referred to as the wet and dry terms. The vertical gradient of potential refractive index, $M$, is further defined as

$$
\begin{aligned}
M & =-77.6 \times 10^{-6} p / T(\partial \ln \theta / \partial z) /\{1+15,500 q / T \\
& \cdot[1-(\partial \ln q / \partial z) /(2 \partial \ln \theta / \partial z)]\} \\
M= & -77.6 \times 10^{-6} p / T\left\{N^{2} / g+15,500 q / T\right. \\
& \left.\cdot\left[N^{2} / g-(\partial \ln q / \partial z) / 2\right]\right\}
\end{aligned}
$$

where $\theta, q$, and $N$ are the potential temperature, specific humidity, and Brunt-Väisälä (buoyancy) frequency, respectively [e.g., Ottersten, 1969; Gage et al., 1985]. Note that since $p / T$ is proportional to air density, the dry term of $M$, i.e., neglecting humidity, is proportional to $N^{2}$ and air density. Therefore $M$ generally decreases with the scale height of air density (approximately $7 \mathrm{~km}$ ) in the stratosphere where the wet term can be neglected and the background value of $N^{2}$ is roughly constant. The measured vertical echo power is expressed as follows:

$$
P_{v}=P_{\imath} \lambda^{2} /\left(16 \pi^{2} r^{2}\right) G^{2} \rho^{2}
$$

where $P_{t}, \lambda, r, G$, and $\rho$ are the transmitted power, radar wavelength, range, antenna gain, and reflection coefficient, respectively.

For the Fresnel reflection the reflection coefficient is assumed to be proportional to $M^{2}$ and the intensity of fluctuations with a vertical scale of half of the radio wavelength $E(2 k)$ [VanZandt and Vincent, 1983; Gage et al., 1985], i.e.,

$$
\rho^{2}=C M^{2} E(2 k)
$$

where $C$ is a constant which is determined by the radar wavelength and the height resolution of the radar sampling volume.

On the other hand, the radar reflectivity is proportional to $N^{-2} M^{2} \varepsilon$ for isotropic turbulent scattering observed by oblique beams pointed sufficiently far from the zenith, where $\varepsilon$ is the eddy dissipation rate within a turbulent layer [Gage et al., 1980]. If we consider $(2 b)$, the radar reflectivity for isotropic turbulent scattering becomes proportional to $N^{2} \varepsilon$ as the humidity goes to zero, while $\rho^{2}$ for specular reflection detected near the vertical direction becomes proportional to $N^{4} E(2 k)$. Therefore these two scattering mechanisms are characterized by proportionality to either $N^{2}$ or $N^{4}$ as well as the turbulence parameters.

In the following sections we will show that variations of vertical echo power are determined mainly by variations of $M^{2}$ rather than variations of $E(2 k)$. Furthermore, we will examine the effects of gravity waves on the structure of the vertical echo power profile.

For convenience we now define a normalized signal-to-noise ratio (SNR) in the vertical direction, $S_{v}$, after compensating for the range-squared effect as follows

$$
S_{v}=\left(P_{v} / P_{N}\right)(r / 10)^{2}
$$

where $P_{N}$ and $r$ are the noise level and the range in kilometers, respectively. The reflection coefficient described in (4) becomes proportional to $S_{v}$. The SNR in the oblique direction $S_{0}$ is also normalized similarly to (5) after compensating for both the rangesquared effect and the decrease of the effective antenna area.

\section{VERTICAL ECHO POWER AND TEMPERATURE PROFILES}

We have monitored the echo power in the troposphere and lower stratosphere with the MU radar by pointing the antenna beam in the vertical as well as in two oblique directions aligned north and east at a zenith angle of $20^{\circ}$. In this study we have mainly used data collected in the vertical direction. Figure 1 shows time-height contours of vertical echo power observed for about 71 hours on September 24-27, 1986. Data were collected at altitudes between 5.4 and $21 \mathrm{~km}$ by oversampling every $75 \mathrm{~m}$ with a height resolution of $150 \mathrm{~m}$ and a time resolution of about 74 s. In this analysis, data were averaged over five records in order to make the entire data set compact.

Below about $10 \mathrm{~km}$ there was a large time-height variation of intense scattering layers. A thin intense echoing layer can be recognized at altitudes of 9-12 $\mathrm{km}$, whose relation to a passage of a high-level front will be discussed in the next section. In the stratosphere, reflection layers are fairly stratified and show downward or upward phase progressions, suggesting a manifestation of gravity wave effects on these reflection layers.

Since temperature and humidity profiles play a major role in determining the characteristics of verti- 


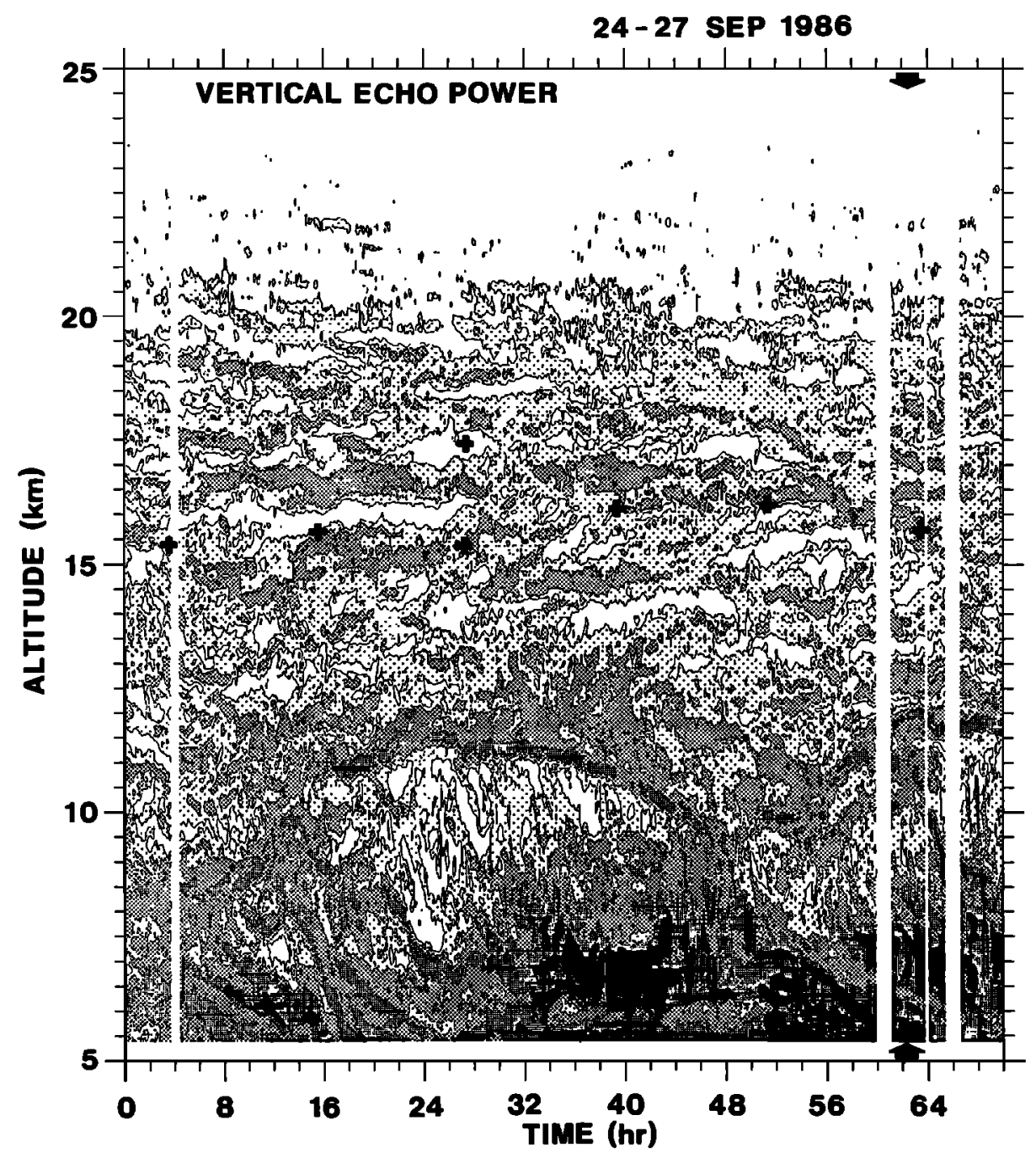

Fig. 1. Contour plot of the vertical echo power detected by MU radar observations starting at 1730 LT on September 24, 1986. A radiosonde was launched at about 60 hours after the start of the MU radar observations, as indicated by a thick arrow. A cross indicates the tropopause height determined from routine radiosonde soundings at Shionomisaki.

cal echo power, we have launched a radiosonde from the MU radar site. By adding a microcomputer system to the data acquisition system of a normal radiosonde, we are able to sample temperature, pressure, and humidity every $1 \mathrm{~s}$ (5-m resolution). We have smoothed these profiles by averaging over $30 \mathrm{~m}$ in order to reduce the quantization error and to obtain equally spaced points from the irregularly spaced original data points. These profiles are further smoothed by using a running mean window over five adjacent points, which gives the temperature profile with the height resolution of $150 \mathrm{~m}$ similar to that for the MU radar observations.
By using a low-pass filter with a cutoff wavelength of $3 \mathrm{~km}$, a smoothed temperature profile, $T_{0}$, is determined as shown in Figure 2. The tropopause was located at around $15 \mathrm{~km}$. The vertical gradient of $T_{0}$ at $10-15 \mathrm{~km}$ altitudes is gradual relative to that in the region below $10 \mathrm{~km}$. Therefore for the purposes of further discussion, altitudes between 10 and $15 \mathrm{~km}$ will be referred to as the upper troposphere.

Residual temperature perturbations $\left(T^{\prime}\right)$ also plotted in Figure 2 have amplitudes ranging up to $2 \mathrm{~K}$ with a dominant vertical wavelength of $2 \mathrm{~km}$ in the stratosphere. By utilizing a unique data set of temperature profiles from the radiosondes launched from 


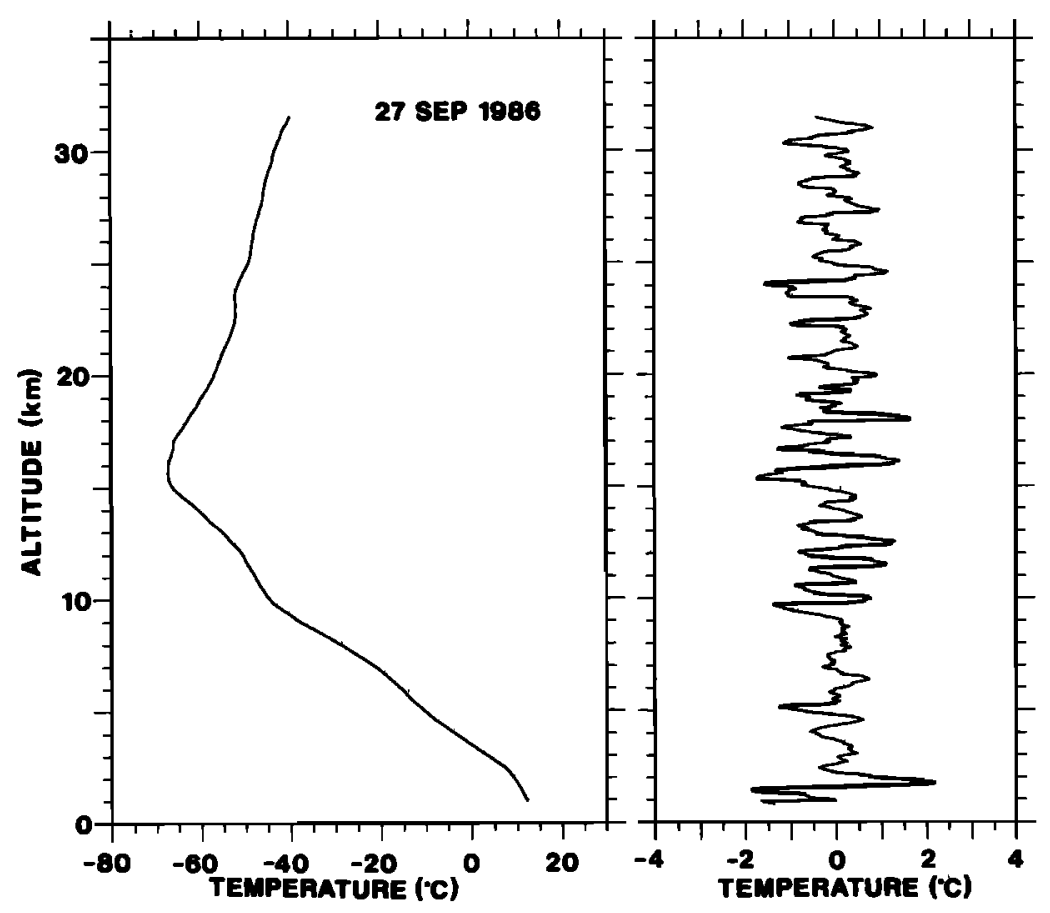

Fig. 2. Background temperature profile after a low-pass filtering with a cutoff of $3.0 \mathrm{~km}\left(T_{0}\right)$ (left) and residual temperature fluctuations $\left(T^{\prime}\right)$ (right) measured by a radiosonde launched from the MU radar site at 0712 LT on September 27, 1986.

the MU radar site as well as wind measurements taken by the MU radar in October and November 1986, Fritts et al. [1988] have extensively studied the characteristics of vertical wave number spectra of these fluctuations. They found excellent agreement between the observed and theoretical saturated vertical wave number spectra of both temperature and wind velocity. Fritts et al. concluded that the mesoscale fluctuations in the temperature and wind velocity fields in the troposphere and lower stratosphere are explained by a saturated wave number spectrum of gravity waves with a slope of -3 at high wave numbers and a break at a dominant vertical wavelength of around $2.5 \mathrm{~km}$. The profile of the perturbation temperature plotted in Figure 2 shows quite similar behavior to that determined by Fritts et al. Therefore we assume here that the temperature fluctuation can be attributed to saturated gravity waves.

Figure 3 shows $S_{v}$ and $S_{0}$ measured during the radiosonde sounding shown in Figure 2 . In the lower stratosphere, aspect sensitivity of the normalized SNR ranging from 10 to $25 \mathrm{~dB}$ exists at all heights, which indicates that Fresnel reflection from stratified layers is dominant in the vertical direction [Tsuda et al., 1986]. On the other hand, in the troposphere there is strong aspect sensitivity in layers with a thickness of $0.5-1 \mathrm{~km}$ separated by relatively narrow non-aspect sensitive regions. At $8.5-9.5 \mathrm{~km}$ the aspect sensitivity becomes fairly small, suggesting predominance of an isotropic turbulent scattering region. The upper troposphere seems to be stratified enough to give intense specular reflections similar to those in the stratosphere.

\section{CHARACTERISTICS OF LOWER TROPOSPHERIC ECHO POWER}

The refractive index gradient in the troposphere includes effects of specific humidity $q$ and its vertical gradient $\partial q / \partial z$. As shown in Figure 4, the specific humidity was large below $1.5 \mathrm{~km}$ altitude. We see that $q$ had an enhancement at $4.5-5.2 \mathrm{~km}$, a local minimum at $5.5-6 \mathrm{~km}$, and a fairly broad enhancement above $6 \mathrm{~km}$ with a sharp ceiling at around 10 $\mathrm{km}$, which corresponds to the bottom of the upper troposphere. Figure 4 also shows a profile of $|15,500 q / T[1-(\partial \ln q / \partial z) /(2 \partial \ln \theta / \partial z)]|$, which corresponds to the absolute ratio between the wet and dry terms from (2a). That is, when this ratio is considerably larger than $1, M$ is dominated by the wet term. The ratio tends to become large at altitudes of 


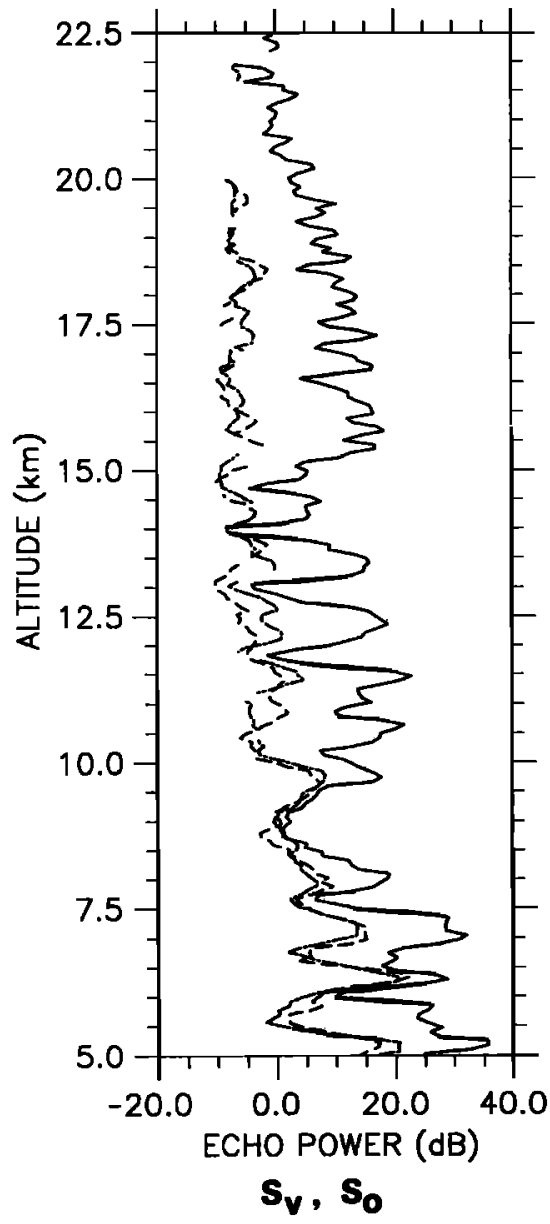

Fig. 3. Normalized SNR $\left(S_{v}\right.$ and $S_{0}$ ) averaged over 45 records (56 min) during the radiosonde measurements shown in Figure 2. Solid, dashed, and dot-dashed lines correspond to profiles detected in the vertical, northward, and eastward directions.

large $q$, and its fine height structure is mainly determined by the $\partial q / \partial z$. At altitudes below about $10 \mathrm{~km}$, humidity enhances $M^{2}$ by 10-20 dB over the dry term, as shown in the right-hand panel.

Echo power profiles collected by the MU radar during the ascent of the radiosonde are averaged over five records, which gives a time resolution of about $6 \mathrm{~min}$. They are then used to calculate the $S_{v}$ profiles shown in Figure 5. In the lower troposphere (below $10 \mathrm{~km}$ ) these $S_{\mathrm{v}}$ profiles have peaks and dips at altitudes corresponding to those of the $M^{2}$ profile, although the intensities of peaks vary rapidly. The mean of these individual $S_{v}$ profiles, which is also plotted in Figure 5, does not agree well with the $M^{2}$ profile in Figure 4 in the lower troposphere. This comparison might suggest that the time-height variation of the humidity profile, which is the major factor in determining the fine structure of the $M^{2}$ profile, is faster than the time resolution of the radiosonde measurements. That is, a radiosonde needs about $30 \mathrm{~min}$ to reach the $10 \mathrm{~km}$ altitude, measuring local humidity at each height, while the MU radar monitors vertical echo power instantaneously. Hence the $\boldsymbol{M}^{2}$ profile in Figure 4 does not necessarily represent the mean profile at a given time; the $M^{2}$ profile might not be stable enough for a proper comparison with the mean $S_{v}$ profile.

On the other hand, the peaks of the $S_{v}$ profiles at around 12.5 and $13.5 \mathrm{~km}$ in the upper troposphere show relatively small variations in their altitudes and peak intensities. The $M^{2}$ profile agrees fairly well with the mean profile of $S_{v}$ at these heights. This suggests that both $S_{v}$ and $M^{2}$ above about $10 \mathrm{~km}$, where the latter is not appreciably affected by the humidity, vary more slowly than the time resolution of radiosonde measurements.

The large time-height variations of echo power in the lower troposphere shown in Figure 1 might suggest a dominance of convective motions in the troposphere. However, we should note that the variation of echo power cannot be directly interpreted as the manifestation of convective motions, but indicates the variability of structure of the humidity profile, especially its vertical gradient.

Below $12 \mathrm{~km}$ near the center of the observing period, intense echoes are received from a thin descending layer as shown in Figure 1. Figure 6 shows a contour plot of $M^{2}$ including only the dry term deduced from routine radiosonde measurements at Shionomisaki weather station about $100 \mathrm{~km}$ south of the MU radar. The sparse temperature profiles sampled every 12 hours are linearly interpolated in order to draw a contour map. The $M^{2}$ contours agree with the behavior of the intense reflection layer at altitudes of $10-12 \mathrm{~km}$. A discrepancy is that the enhanced layer of $M^{2}$ in Figure 6 only descends to about $10 \mathrm{~km}$, while the intense echoing layer continues to descend to about $9.5 \mathrm{~km}$. This is most likely an artifact of the poor time resolution (12 hours) of the routine soundings, so that the final part of the descent of the layer was missed.

The region of the intense echo also corresponds to a region of large vertical shear in the measured horizontal wind. This indicates that there are large horizontal temperature gradients as well as a large vertical gradient. We may therefore identify this as a baroclinic zone, i.e., an upper level front. Some of the weaker layers observed in the troposphere in Figure 1 also correspond to regions of strong wind shear. 

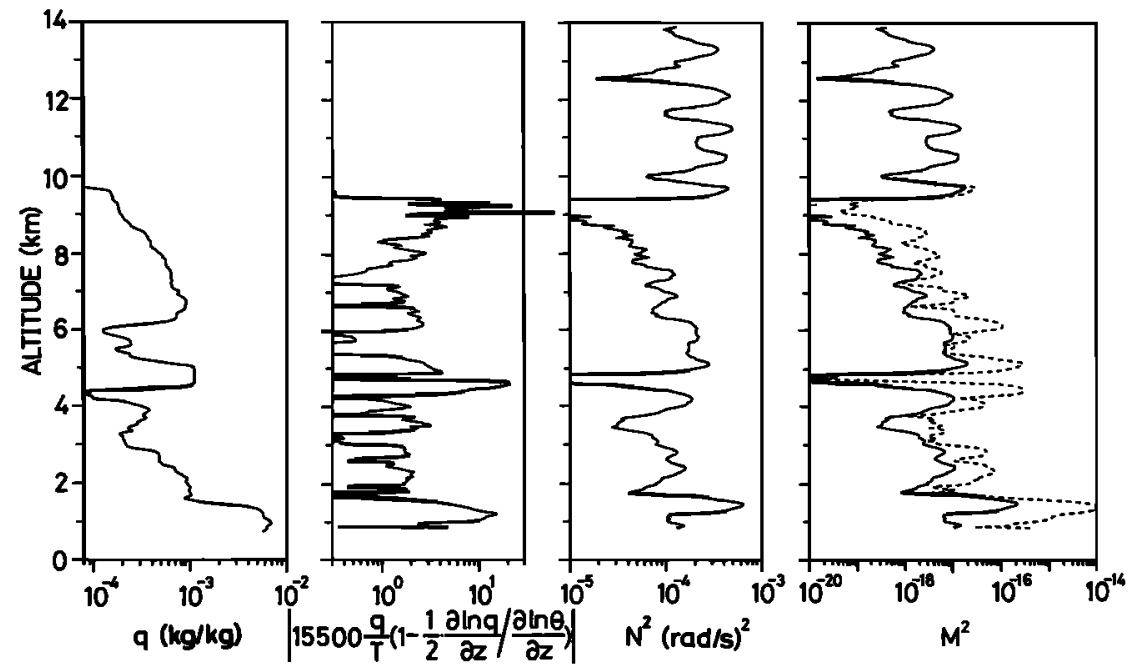

Fig. 4. Profiles of specific humidity (left), $|15,500 q / T[1-(\partial \ln q / \partial z) /(2 \partial \ln \theta / \partial z)]|$ (second panel from left), $N^{2}$ (third panel from left), and $M^{2}$ (right) deduced from radiosonde data taken at the MU radar site. The vertical spacing for the calculation of derivatives is $150 \mathrm{~m}$. Solid and dashed lines in the right panel correspond to $M^{2}$ profiles with and without humidity, respectively.

\section{REFLECTION ECHOES IN THE UPPER TROPOSPHERE AND STRATOSPHERE}

Figure 7 shows $N^{2}$ and $M^{2}$ profiles measured between 10 and $20 \mathrm{~km}$, where the solid and dashed curves are determined by using vertical spacings of $150 \mathrm{~m}$ and $30 \mathrm{~m}$ for the calculation of temperature gradients. These two profiles of $M^{2}$ are hereafter referred to as $M_{150}^{2}$ and $M_{30}^{2}$, respectively. Because $M_{150}^{2}$ has the same height resolution as the MU
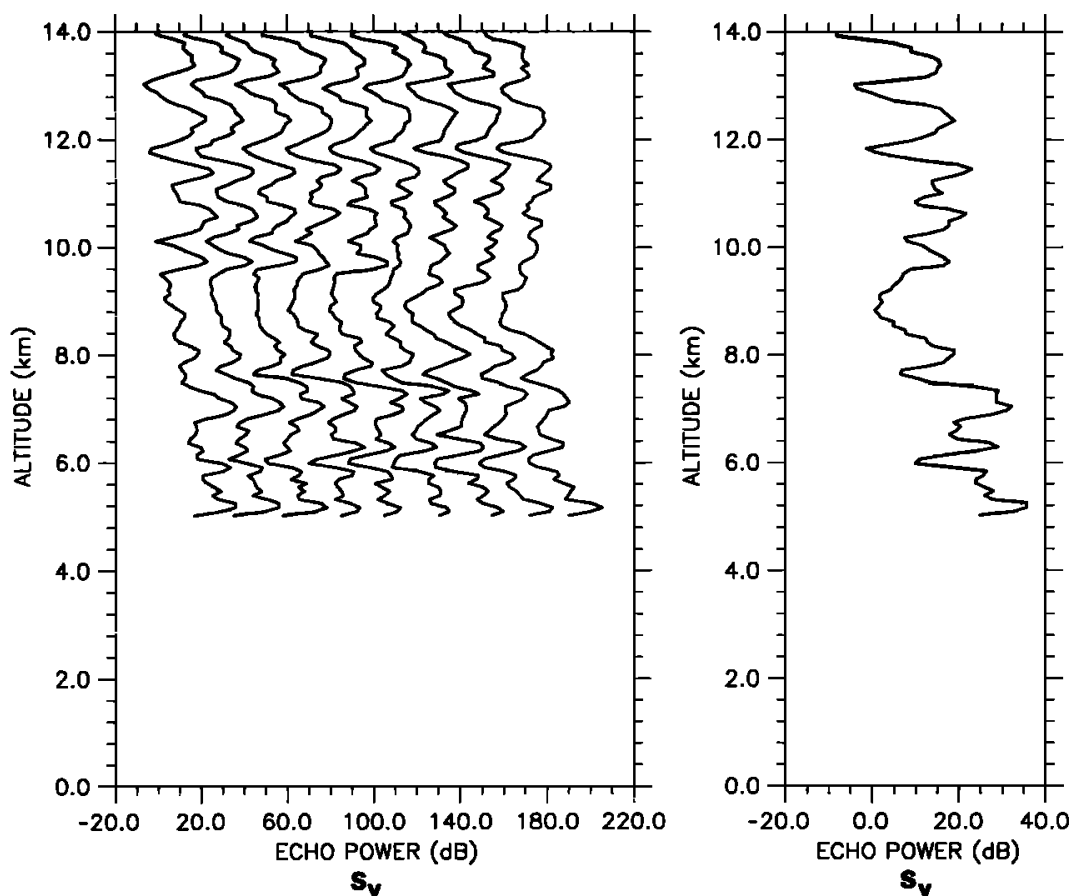

Fig. 5. Profiles of $S_{v}$ averaged over $6 \mathrm{~min}$ by the MU radar (left), where successive profiles are displaced by $20 \mathrm{~dB}$.

The mean profile averaged over nine determinations is also plotted in the right panel. 


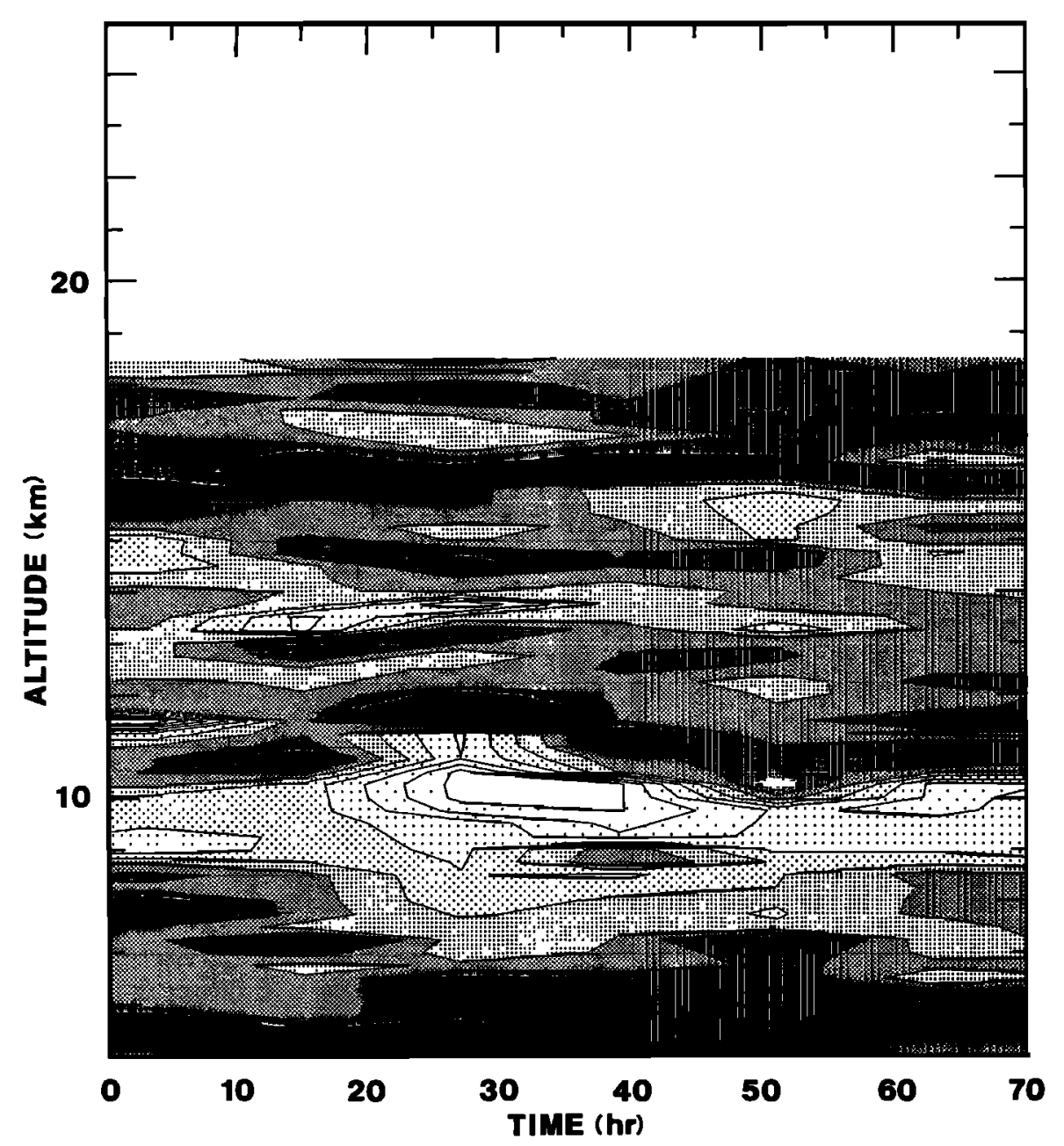

Fig. 6. Contour plot of $M^{2}$ including only the dry term deduced from radiosonde soundings of temperature at Shionomisaki weather station located about $100 \mathrm{~km}$ south of the MU radar. Contour lines are plotted every $3 \mathrm{~dB}$.

radar observations of echo power, it is mainly used in this section.

Because the effect of humidity is negligible in the upper troposphere (above $10 \mathrm{~km}$ ) and stratosphere, $M$ becomes proportional to air density and $N^{2}$ as described in $(2 b)$. Since relative fluctuations of air density are of an order of $\left(T^{\prime} / T_{0}\right)$, which is less than $1 / 100$ as shown in Figure 2, the vertical structure of $M$ is mainly determined by the $N^{2}$ profile. Indeed, the fine structure of the $M_{150}^{2}$ profile plotted in Figure 7 is very similar to that of $\left(N^{2}\right)^{2}$, although there is a gradual attenuation of $M_{150}^{2}$ relative to the $\left(N^{2}\right)^{2}$ profile with the scale height of atmospheric density. Peak values of $M_{150}^{2}$ range from $10^{-17}-10^{-18}$, and a typical vertical spacing of these peaks is $1-2 \mathrm{~km}$ in this height range.

The mean profile of $S_{v}$ also plotted in Figure 7 shows peaks with similar vertical spacings as those in the $M_{150}^{2}$ profile below about $15 \mathrm{~km}$ and slightly smaller spacings above $15 \mathrm{~km}$. The ratio of $S_{v}$ between the peaks and valleys is about 20 and $10 \mathrm{~dB}$ below and above $15 \mathrm{~km}$, respectively, which is also consistent with the range of variation of $M_{150}^{2}$.

If the $M_{150}^{2}$ and $M_{30}^{2}$ profiles are compared with each other, it can be recognized that peaks of $M_{150}^{2}$ with thickness of $0.5-1 \mathrm{~km}$ consist of several thinner peaks of $M_{30}^{2}$. Some peaks of the $S_{v}$ profile generally correspond to those of $M_{30}^{2}$, although the agreement is not perfect. It might be suggested that the $M^{2}$ profile, when it is measured with very good height resolution, consists of many peaks and valleys with vertical spacing smaller than the radar height resolution $(150 \mathrm{~m})$. Thus the vertical echo received by the radar might be an incoherent summation of reflec- 


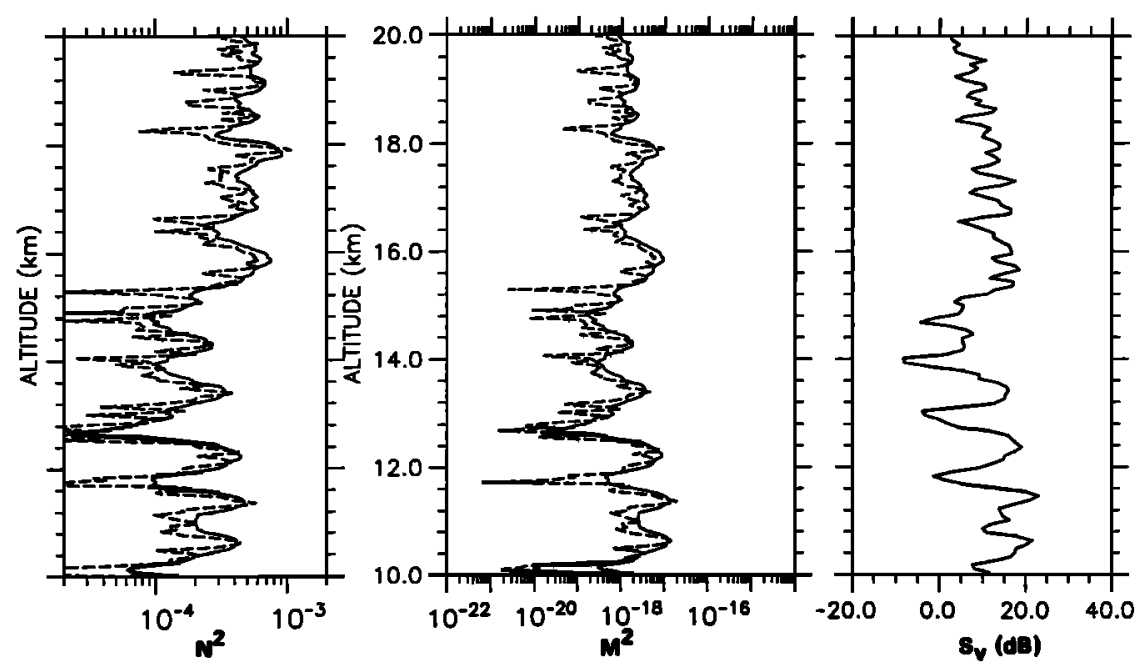

Fig. 7. Vertical profiles of $N^{2}$ (left) and $M^{2}$ (center) deduced from the radiosonde measurement, along with the $S_{v}$ profile averaged over $56 \mathrm{~min}$ of the MU radar observations (right). Solid and dashed lines are calculated from derivatives with vertical spacings of 150 and $30 \mathrm{~m}$, respectively.

tions contributed from these thin local enhancements of $M^{2}$, which is not inconsistent with the fundamental idea of volume scattering assumed in deriving (4) [Van Zandt and Vincent, 1983; Gage et al., 1985].

In order to exhibit more precisely the similarity between $S_{v}$ and $M_{150}^{2}$ profiles, these are replotted together in Figure $8 a$. Below $14 \mathrm{~km}$ these profiles agree quite well except for differences in intensity at a valley of about $11.8 \mathrm{~km}$ altitude and a discrepancy of the altitude of a valley near $13 \mathrm{~km}$. At altitudes of 14-15 km, which correspond to the region just below the tropopause, $S_{v}$ is attenuated in relation to $M_{130}^{2}$ by 5-10 dB more than in other altitude regions. In the height range from 15 to $18 \mathrm{~km}, S_{v}$ and $M^{2}$ generally show similar height structures, although the $M_{1 \text { so }}^{2}$ profile is smoother. Above $18 \mathrm{~km}, S_{v}$ tends to decrease more rapidly than $M_{150}^{2}$. The ratio between $S_{v}$ and $M_{150}^{2}$ plotted in Figure $8 b$ shows a variation ranging from -15 to $15 \mathrm{~dB}$ from the mean value. However, the large excursion from the mean mostly occurs at valleys of $S_{v}$, where estimation of echo power is less reliable than at peaks. The spacial variation of $M^{2}$ might also be the cause of the excursion, because the horizontal distance between the MU radar and the radiosonde could become as large as several tens of kilometers at these altitudes. A solid circle in Figure $8 b$ corresponds to a determination at a peak of $S_{v}$ whose location is indicated by a short horizontal bar in Figure 8a. The mean value of the ratio at the peaks is $189.5 \mathrm{~dB}$ with a standard deviation of $2.4 \mathrm{~dB}$ indicated as straight vertical lines in Figure $8 b$.
A linear regression or a cross-correlation analysis between $\log \left(M_{1}^{2}\right.$ so $)$ and $\log \left(S_{v}\right)$ gives a quantitative idea about the relation between these two profiles. Since the measurement accuracy at $M_{150}^{2}$ does not seem to be affected by the intensity of $M_{1 \text { so }}^{2}$ itself, and on the other hand, the determination of $S_{v}$ becomes worse when the SNR is small, we have assumed $\log \left(M_{150}^{2}\right)$ as the independent variable and $\log \left(S_{v}\right)$ the dependent variable in the linear regression analysis plotted in Figure $8 c$. The slope of the linearly fitted curve is 0.98 with a standard deviation of 0.1 for the range of $M_{1 \text { so }}^{2}$ larger than $-183 \mathrm{~dB}$. The cross-correlation value between $M_{1 \text { so }}^{2}$ and $S_{v}$ for the entire range of variables is about 0.7 as shown in Figure 8d.

These analyses indicate that the height structure of $S_{v}$ with a vertical resolution of $150 \mathrm{~m}$ is mainly determined by $M^{2}$, thus $N^{2}$, which suggests that in (4), $M^{2}$ varies much more than the factor $E(2 k)$.

As described concerning the profile of temperature perturbation shown in Figure 2, Fritts et al. [1988] have shown that at wave numbers greater than the breakpoint (around 1/2.5 c/km) the vertical wave number spectra of both temperature and wind velocity have a slope of -3 , which quantitatively agrees well with the saturated spectrum of gravity waves. We will now investigate a slope of the vertical wave number spectra of $N^{2}$ at higher wave numbers than the breakpoint. If temperature perturbations are expressed by a Fourier series, then the spectral energy density of $N^{2}$ is proportional to the product of the wave number squared and the spectral energy den- 

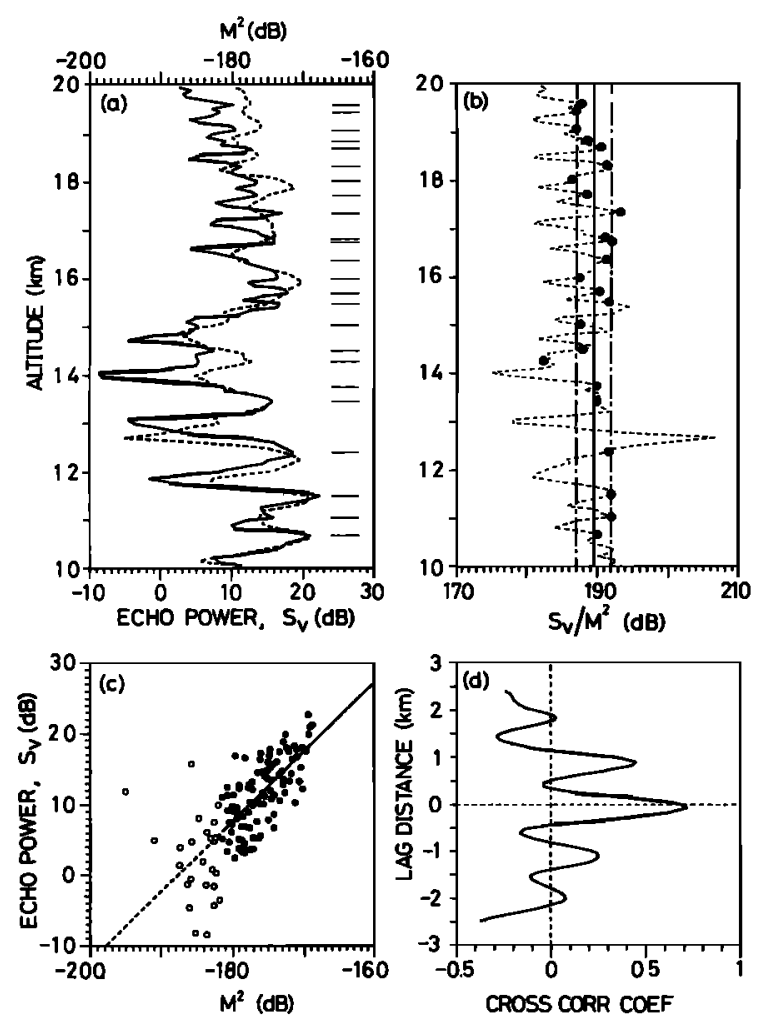

Fig. 8. Comparison analyses between $M_{150}^{2}$ and $S_{v}:(a)$ simultaneous plot, where solid and dashed lines correspond to $S_{v}$ and $M_{150}^{2}$, respectively, (b) ratio, (c) a linear regression curve, and (d) cross-correlation coeficient.

sity of temperature perturbations, because $N^{2}$ is calculated from the vertical derivative of the temperature perturbation. Therefore a slope of -3 for temperature perturbations is transformed into a slope of -1 for $N^{2}$ spectra.

By using the same data analysis technique used by Fritts et al., we have calculated the vertical wave number spectra of the normalized temperature perturbation and $N^{2}$ observed on September 27, 1986. The results are plotted in Figure 9 in normal and area-preserving forms. The spectral density fluctuates significantly owing to daily variability of gravity wave activity as also detected in their spectra by Fritts et al. The theoretical saturated spectrum discussed by Fritts et al. predicts both the slope $(-1)$ and amplitude of the temperature spectrum. This theoretical line, also plotted in Figure 9, agrees well with the observed temperature spectrum at wave numbers larger than the breakpoint of $5 \times 10^{-4} \mathrm{c} / \mathrm{m}$. Spectral densities of the peaks in the $N^{2}$ spectra near 6 $\times 10^{-4}, 1.1 \times 10^{-3}$, and $1.6 \times 10^{-4} \mathrm{c} / \mathrm{m}$ decrease

roughly following a slope of -1 , which is consistent with the investigation described above.

The area-preserving graph of temperature perturbation indicates that the energy of the temperature perturbations is concentrated around the dominant vertical scale (breakpoint) of $2 \mathrm{~km}$. On the other hand, the energy of $N^{2}$ spreads over wavelengths smaller than the dominant vertical scale. When spectra of the temperature perturbations have a slope exactly equal to -3 as was found by Fritts et al. [1988] after averaging many spectra, so that the slope of $N^{2}$ spectra becomes -1, the area-preserving graph of $N^{2}$ spectra becomes constant at wave numbers larger than the breakpoint. This means that the energy (variance) of $N^{2}$ perturbations integrated over one decade of wave numbers is the same in any wave number range greater than the breakpoint.

The echo power is proportional to $M^{2}$, thus to $\left(N^{2}\right)^{2}$. The distribution of spectral density of $\left(N^{2}\right)^{2}$ can be estimated from a simple relation of sinusoidal functions. We assume that the $N^{2}$ perturbations are again expressed by Fourier series and that the Fou-
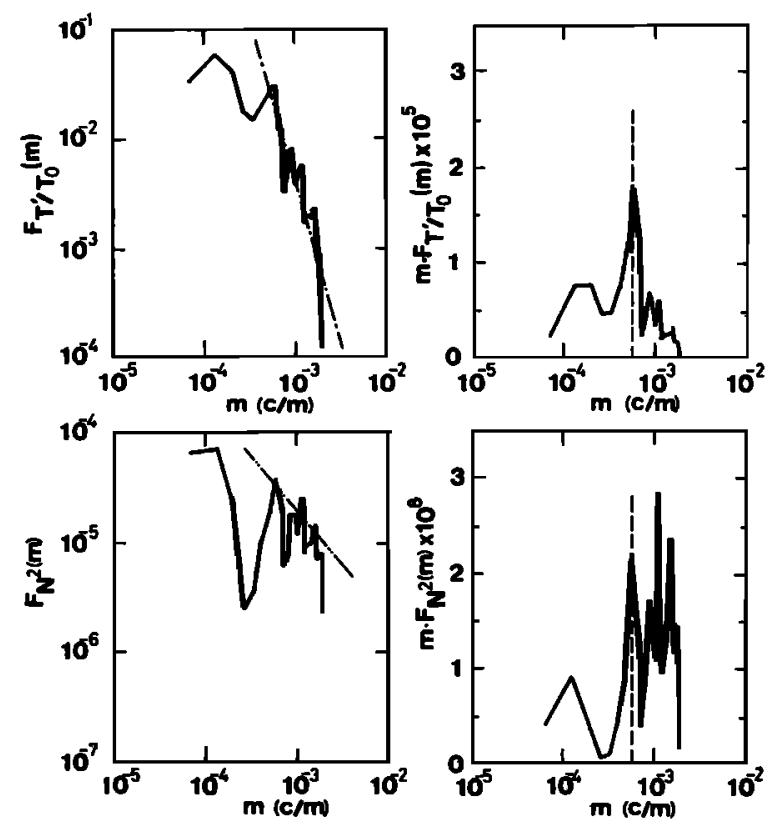

Fig. 9. Vertical wave number spectra of normalized temperature perturbation (top panels) and Brunt-Väisälä frequency squared (bottom panels). Left panels show normal spectra, and right panels show area-preserving graphs in which the integrated area under each spectral curve is directly proportional to the integrated energy. Dot-dashed lines in the top left and bottom left panels show slopes of -3 and -1 , respectively. Vertical dashed lines in the right panels indicate the dominant wavelength of approximately $\mathbf{2} \mathbf{~ k m}$ for temperature perturbations. 
rier components are independent of each other. Then, by squaring each Fourier component, its energy density is transferred to the wave number a factor of 2 larger than the original wave number. Therefore the dominant vertical spacings of the echo power profile are biased toward smaller scales than the dominant vertical wavelength of the fluctuations in temperature and wind fields. Since the echo power profile correlates with the $M^{2}$ profile, the richness of the $M^{2}$ profile on smaller scales suggests that the echo power profile may also be rich on smaller scales. This would be consistent with the discussion of the correlation between the echo power and $M_{30}^{2}$ profiles shown in Figure 7.

In summary, we have experimentally found that the vertical structure of stratospheric vertical echo power is mainly determined by $\boldsymbol{M}^{2}$ and, therefore, by $\left(N^{2}\right)^{2}$ considering (2), (3), and (4). Fluctuations of $N^{2}$ seem to be affected by gravity waves through their effects on the temperature field.

\section{CONCLUSION}

We have presented simultaneous observations of vertical echo power in the troposphere and lower stratosphere detected by the MU radar and the refractive index gradient measured by a radiosonde. In the troposphere the wet term gives an enhancement of $M^{2}$ by about $10-20 \mathrm{~dB}$ over the dry term, although the overall height structure of $M^{2}$ is similar to the dry term. The vertical gradient of humidity generates fine structure in the $M^{2}$ profile such as sharp peaks and deep dips. Large time-height variations of tropospheric echo power can be attributed to rapid changes in the humidity profile, which may be affected by convective motions. We have observed the passage of a high-level front over the MU radar and also its effects on refractive index gradient through perturbations in the temperature field.

In the upper troposphere and stratosphere the fine vertical structure of $M^{2}$ is mainly determined by the Brunt-Väisälä frequency. We have found excellent agreement between the $S_{v}$ and the $M^{2}$ profiles with a radar range resolution of $150 \mathrm{~m}$. A linear regression analysis also shows that $S_{v}$ is proportional to $M^{2}$. This indicates that intense peaks in the vertical echo power profiles represent the local enhancement of Brunt-Väisälä frequency probably induced by gravity waves through their modification of the temperature field. Unfortunately, we cannot determine the origin of the refractive index fluctuations with a scale of $\mathbf{3 ~ m}$ (half of the radar wavelength) from our observations.
However, the linear relation between $S_{v}$ and $M^{2}$ shown in Figure 8 suggests that the vertical structure of the reflection coefficient with height resolution of the MU radar $(150 \mathrm{~m})$ is mainly determined by $M^{2}$ and, therefore, the energy density of 3-m scale fluctuations $E(2 k)$ seems to be distributed rather uniformly with height, although $E(2 k)$ is certainly another important factor to determine the intensity of the reflection coefficient.

Vertical wave number spectra of temperature and Brunt-Väisälä frequency agree well with the saturated spectrum of gravity waves with a dominant scale of a few kilometers and slopes of -3 and -1 , respectively, as shown in Figure 9. From an investigation of area-preserving graphs of these spectra, we have found that the spectra of Brunt-Väisälä frequency profiles have large energy at wavelengths smaller than the dominant scale for temperature perturbation, although the energy of the temperature fluctuations is concentrated near the dominant scale. From MU radar observations with good height resolution, we have shown that the vertical spacings of intense reflection layers in the stratosphere, which generally range from $500 \mathrm{~m}$ to a few kilometers, correspond to the dominant vertical scale of fluctuations in the Brunt-Väisälä frequency profile.

Acknowledgments. The authors wish to thank T. E. VanZandt, W. K. Hocking, and R. A. Vincent for valuable suggestions and careful reading of the manuscript. One of the authors (P.T.M.) is supported by a Japan Society for the Promotion of Science postdoctoral fellowship. The MU radar is operated by Radio Atmospheric Science Center, Kyoto University.

\section{REFERENCES}

Bean, B. R., and E. J. Dutton, Radio Meteorology, p. 431, U. S. Government Printing Office, Washington, D. C., 1966.

Fritts, D. C., T. Tsuda, T. Sato, S. Fukao, and S. Kato, Observational evidence of a saturated gravity wave spectrum in the troposphere and lower stratosphere, J. Atmos. Sci., in press, 1988.

Fukao, S., T. Sato, T. Tsuda, S. Kato, K. Wakasugi, and T. Makihira, The MU radar with an active phased array system, 1, Antenna and power amplifiers, Radio Sci., 20, 1155-1168, 1985a.

Fukao, S., T. Tsuda, T. Sato, S. Kato, K. Wakasugi, and T. Makihira, The MU radar with an active phased array system, 2, In-house equipment, Radio Sci., 20, 1169-1176, 1985 b.

Gage, K. S., and B. B. Balsley, On the scattering and reflection mechanisms contributing to clear air radar echoes from the troposphere, stratosphere, and mesosphere, Radio Sci., 15, 243$257,1980$.

Gage, K. S., and J. L. Green, Evidence for specular reflection from monostatic VHF radar observations of the stratosphere, Radio Sci., 13, 991-1001, 1978.

Gage, K. S., J. L. Green, and T. E. VanZandt, Use of Doppler radar for the measurement of atmospheric turbulence parame- 
ters from the intensity of clear air echoes, Radio Sci., 15, 407$416,1980$.

Gage, K. S., W. L. Ecklund, and B. B. Balsley, A modified Fresnel scattering model for parameterization of Fresnel returns, Radio Sci., 20, 1493-1501, 1985.

Hocking, W. K., and J. Röttger, Pulse length dependence of radar signal strengths for Fresnel backscatter, Radio Sci., 18, 13121324, 1983.

Kato, S., T. Ogawa, T. Tsuda, T. Sato, I. Kimura, and S. Fukao, The middle and upper atmosphere radar: First results using a partial system, Radio Sci., 19, 1475-1484, 1984.

Ottersten, H., Mean vertical gradient of potential refractive index in turbulent mixing and radar detection of CAT, Radio Sci., 4, 1247-1249, 1969.

Röttger, J., Reflection and scattering of VHF radar signals from atmospheric reflectivity structures, Radio Sci., 15, 259-276, 1980.

Röttger, J., and C. H. Liu, Partial reflection and scattering of VHF radar signals from the clear atmosphere, Geophys. Res. Lett., 5, 357-360, 1978.
Tsuda, T., T. Sato, K. Hirose, S. Fukao, and S. Kato, MU radar observations of the aspect sensitivity of backscattered VHF echo power in the troposphere and lower stratosphere, Radio Sci., 21, 971-980, 1986.

VanZandt, T. E., and R. A. Vincent, Is VHF Fresnel reflectivity due to low-frequency buoyancy waves?, in Handbook for MAP, vol. 9, pp. 78-80, Middle Atmosphere Program, University of Illinois, Urbana, 1983.

VanZandt, T. E., J. L. Green, K. S. Gage, and W. L. Clark, Vertical profiles of relfectivity turbulence structure constant: Comparison of observations by the Sunset radar with a new theoretical model, Radio Sci., 13, 819-829, 1978.

S. Fukao, S. Kato, P. T. May, T. Sato, and T. Tsuda, Radio Atmospheric Science Center, Kyoto University, Uji, Kyoto 611, Japan. 